Check for updates

Cite this: Food Funct., 2019, 10, 3135

Received 14th February 2019,

Accepted 18th April 2019

DOI: $10.1039 /$ c9fo00298g

rsc.li/food-function

\section{Tissue deconjugation of urolithin A glucuronide to free urolithin $A$ in systemic inflammation $\uparrow$}

\author{
M. A. Ávila-Gálvez, (D) J. A. Giménez-Bastida, (D) A. González-Sarrías (ID) and \\ J. C. Espin (D)*
}

\begin{abstract}
Urolithin A (Uro-A) is an anti-inflammatory and cancer chemopreventive metabolite produced by the gut microbiota from the polyphenol ellagic acid. However, in vivo conjugation of Uro-A to UroA glucuronide (Uro-A glur) dramatically hampers its activity. We describe here for the first time the tissue deconjugation of Uro-A glur to Uro-A after lipopolysaccharide (LPS)-induced inflammation, which could explain the systemic in vivo activity of free Uro-A in microenvironments subjected to inflammatory stimuli.
\end{abstract}

\section{Introduction}

Urolithins are not strictly speaking dietary compounds but catabolic products of ellagitannin (ET) and ellagic acid (EA) metabolism carried out by the gut microbiota. ${ }^{1}$ The most relevant urolithin is urolithin A (Uro-A), a non-toxic metabolite ${ }^{2}$ that is the final urolithin produced by those subjects belonging to the so-called metabotype-A. ${ }^{3}$ Given the extensive and heterogeneous metabolism of ETs and EA in humans, our pioneering studies on urolithins suggested that the plausible in vivo active metabolites after ET and EA consumption could be urolithins. ${ }^{4}$ To date, many studies have reported a plethora of in vitro activities, ${ }^{1,5}$ but the causality of urolithins in the systemic in vivo activity observed after ET and(or) EA-rich food consumption has not yet been unequivocally proven. ${ }^{6}$ Mounting evidence has demonstrated the anti-inflammatory activity, ${ }^{7}$ and the preservation of the gut barrier integrity ${ }^{7,8}$ in murine models after oral Uro-A administration, which is consistent with the fact that the colon is the organ where the highest concentration of urolithins occurs. ${ }^{9}$ Remarkably, Ryu et al. ${ }^{10}$ reported the increase of muscle function in mice after oral Uro-A administration, but the specific metabolite involved in such an effect in tissues remains elusive.

Laboratory of Food \& Health, Research Group on Quality, Safety and Bioactivity of Plant Foods, Department of Food Science and Technology, CEBAS-CSIC, P.O. Box 164, 30100 Campus de Espinardo, Murcia, Spain. E-mail: jcespin@cebas.csic.es $\dagger$ Electronic supplementary information (ESI) available. See DOI: 10.1039/ c9fo00298g
The phase-II metabolism severely hampers the systemic activity of urolithins, including Uro-A. Oral Uro-A bioavailability and tissue distribution are very low. ${ }^{11,12}$ Circulating free Uro-A concentration is negligible since it is extensively conjugated to yield mainly Uro-A glucuronide (Uro-A glur) derivatives in humans. ${ }^{1,5,13}$ In contrast to the relevant in vitro activity of Uro-A, conjugated derivatives show much lower activity. ${ }^{14-17}$

The low bioavailability, extensive phase-II metabolism and gut microbiota catabolism are common features of dietary phenolic compounds. While the health benefits upon consumption of polyphenols have been widely reported, ${ }^{18-21}$ the identification of the final metabolites involved in such effects, especially in systemic tissues, is still under debate. Although some conjugated metabolites (mainly glucuronides and sulphates) can exert certain in vitro activity, ${ }^{14,22-24}$ the direct involvement of these metabolites in the in vivo effects has not yet been fully demonstrated. A plausible explanation for this apparent paradox is the so-called 'deconjugation in inflammation, ${ }^{25,26}$ which has been evidenced in vivo in the case of luteolin, ${ }^{25}$ curcumin, ${ }^{27}$ and especially in quercetin. ${ }^{28-32}$

In the present study, our objective was to explore the 'deconjugation in inflammation' hypothesis in the case of urolithins. To illustrate this, we evaluate here for the first time the tissue deconjugation of Uro-A glur to free Uro-A after oral UroA administration in a rat model of systemic inflammation induced by lipopolysaccharide (LPS).

\section{Materials and methods}

\section{Materials}

Urolithin A (Uro-A) and derived conjugated metabolites (purity $>$ 98\%) were obtained as described elsewhere. ${ }^{33}$ Lipopolysaccharides (LPS) from Escherichia coli serotype O111: B4 were purchased from Sigma-Aldrich (St Louis, MO, USA). Ketamine (Imalgene 1000) was obtained from Merial Laboratories (Barcelona, Spain). All chemicals and reagents were of analytical grade. Formic acid and $\mathrm{HCl}$ were purchased 
from Panreac (Barcelona, Spain). Organic solvents were obtained from Merck (Darmstadt, Germany), and Milli-Q ultrapure water from Millipore Corp. (Bedford, MA, USA).

\section{Animals and experimental design}

The animal ethics committee (University of Murcia, Spain) and the local government (reference A13180503) approved the study. The experimental protocol followed the Directive of the European Council 63/2010/UE, and the Spanish government (RD 53/2013). Male Sprague-Dawley rats $(230-250 \mathrm{~g}$ ) were provided by the Experimental Animal Facility of the University of Murcia. 3-4 rats were housed in a cage in a room with controlled temperature $\left(22 \pm 2{ }^{\circ} \mathrm{C}\right), 55 \pm 10 \%$ relative humidity, and a $12 \mathrm{~h}$ light-dark cycle. Animals were fed with a rat standard chow (Panlab, Barcelona, Spain). Diet and tap water were administered ad libitum until the start of the experiments.

ESI Fig. $1 \uparrow$ shows the design of the experiments. In order to reduce the number of animals in the final experiment, we first explored the maximum plasma glucuronidase activity after intraperitoneal (i.p.) administration of LPS dissolved in phosphate saline buffer (PBS) $(20 \mathrm{mg} \text { per } \mathrm{kg} \text { body weight, b.w. })^{34}$ $(n=3)$ (ESI Fig. 1A $\dagger$ ). The humane endpoint was determined at $5 \mathrm{~h}$ after LPS administration. ${ }^{34}$ Besides, we also determined the plasma pharmacokinetics of Uro-A and derived metabolites after oral Uro-A administration $(n=3)$ (ESI Fig. 1B $\dagger$ ) (26 mg per kg b.w.; equivalent to $250 \mathrm{mg}$ in a $70 \mathrm{~kg}$ person). ${ }^{35}$

The final intervention lasted for 8 hours, in which Uro-A was orally administered to 14 rats by gavage. Three hours after oral Uro-A administration, one group $(n=7)$ served as the control and the other group $(n=7)$ received LPS via i.p. (ESI Fig. 1C $\dagger$ ).

At the end of all interventions (both preliminary and final assays), animals were anaesthetised with ketamine $\left(50 \mathrm{mg} \mathrm{kg}^{-1}\right)$ and sacrificed by cervical dislocation followed by exsanguination.

\section{Sampling procedure}

Blood samples, collected in EDTA-treated tubes, were obtained from the tail vein at $0,0.5,1,2,3,4$ and 5 hours after i.p. LPS administration in the preliminary experiment, and at $0,0.5,1$, 2, 5, 7 and 8 hours after administration by gavage of an aqueous Uro-A suspension in both preliminary and final interventions (ESI Fig. $1 \dagger$ ).

At sacrifice, lungs, kidneys, liver, spleen, prostate, and urinary bladder were removed and snap frozen in liquid nitrogen. The contents of the stomach, small intestine, caecum, and colon were also collected and frozen until further analyses. Urine samples were obtained at sacrifice directly from the bladder and fresh faeces from the distal colon.

\section{Sample processing}

Blood was immediately centrifuged at $14000 \mathrm{~g}$ for $15 \mathrm{~min}$ at $4{ }^{\circ} \mathrm{C}$ in a Sigma 1-13 microcentrifuge (Braun Biotech. International, Germany). The corresponding plasma samples were extracted with acetonitrile : formic acid $(98: 2, \mathrm{v} / \mathrm{v})$, centrifuged, and the supernatant was reduced to dryness in a speed vacuum concentrator. The evaporated samples were re-suspended in methanol, filtered through a $0.22 \mu \mathrm{m}$ polyvinylidene fluoride (PVDF) filter and injected in the UPLC-ESI-QTOF-MS equipment.

Urine samples were centrifuged, filtered through a $0.22 \mu \mathrm{m}$ PVDF filter and diluted with acidified water ( $0.1 \%$ formic acid) before analysis by UPLC-ESI-QTOF-MS.

Lungs, kidneys, liver, spleen, bladder and prostate were extensively washed with PBS to avoid external blood contamination. Tissue samples, as well as the lumen content of the stomach, small intestine, colon and faeces, were processed as previously reported. ${ }^{13,36}$

\section{Analysis of urolithins}

Analyses were performed on an Agilent 1290 Infinity UPLC system coupled to a 6550 Accurate-Mass quadrupole-time-offlight (QTOF) mass spectrometer (Agilent Technologies, Waldbronn, Germany) using an electrospray interface (Jet Stream Technology). Previously validated methods for the analysis of urolithins in urine, plasma and tissues were used. ${ }^{9,33}$ Free urolithins and their conjugated metabolites were identified and quantified in negative mode by peak area integration of their extracted ion chromatograms (EICs) using available standards. The method was validated for the recovery of metabolites, linearity, precision, accuracy, limits of detection (LOD) and quantification (LOQ) as well as for matrix effects as recently reported. ${ }^{17}$

\section{Analysis of $\beta$-glucuronidase activity}

Plasma $\beta$-glucuronidase activity was evaluated by measuring the hydrolysis of 4-methylumbelliferyl-glucuronide to the fluorescent molecule 4-methylumbelliferone as described elsewhere. ${ }^{26}$

\section{Statistical analysis}

Data were analysed using the SPSS Software 24.0 (SPSS Inc., Chicago, IL, USA). The normality of data distribution was tested with the Shapiro-Wilk test. Pharmacokinetics was evaluated using the software PKSolver, ${ }^{37}$ an add-in programme for pharmacokinetic and pharmacodynamic data analysis in MS Excel (Microsoft, Redmond, WA, USA). Pharmacokinetic parameters and differences in the amount of each metabolite between the control and LPS-treated rats in tissues, lumen contents, urine, and blood were compared using either the MannWhitney $U$ test or the two-tailed $t$-test. Differences in the Uro-A glur/free Uro-A ratios between the control and LPS-treated rats were analysed using the Mann-Whitney $U$ test. Results are shown as the mean \pm standard deviation (SD). Significant differences were set at ${ }^{*} P<0.05,{ }^{*} P<0.01$ and ${ }^{* * *} P<0.001$. A trend toward significance was acknowledged when $0.1>{ }^{\#} P>$ 0.05. Data plots were created using Sigma Plot 13.0 (Systat Software, San Jose, CA, USA).

\section{Results}

\section{Effect of LPS on the oral pharmacokinetics of Uro-A}

We first determined the kinetics of LPS-induced plasma $\beta$-glucuronidase. Maximum activity was reached after $2 \mathrm{~h}$ of 


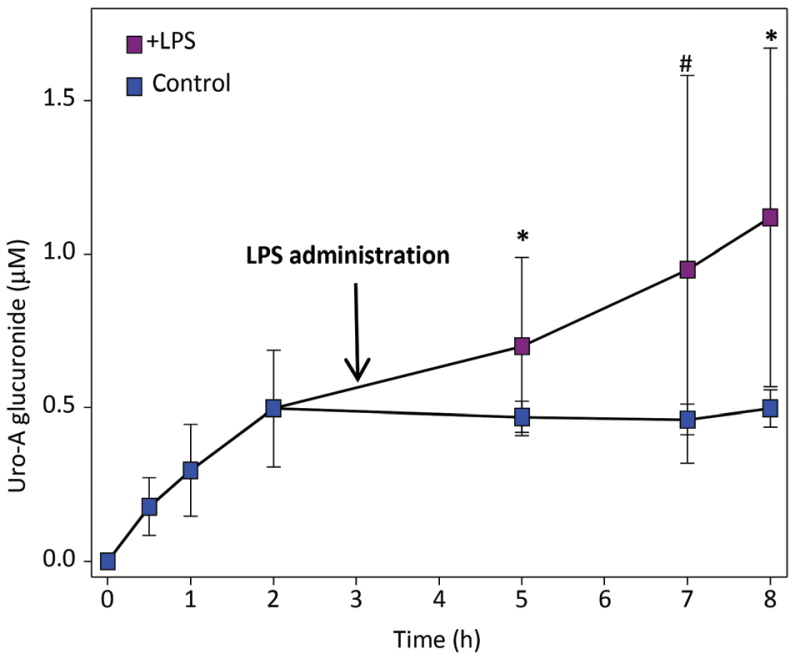

Fig. 1 Plasma pharmacokinetics of Uro-A glur after oral Uro-A administration. Mean values \pm SD are shown. $\square$ Control; $\square$ LPS-treated rats. $0.1>$ ${ }^{\#} P>0.05 ;{ }^{*} P<0.05$.

LPS administration and remained approximately constant up to $5 \mathrm{~h}$, the previously defined endpoint (ESI Fig. $2 \dagger$ ). Next, we investigated the oral pharmacokinetics of Uro-A. The main circulating metabolite detected was Uro-A glucuronide (Uro-A glur) (Fig. 1) and, to a lesser extent, Uro-A sulphate (Uro-A sul) (ESI Fig. $3 \dagger$ ). We could not accurately identify whether the glucuronic moiety was either at the 3- or 8-position in the Uro-A molecule or maybe there was a mixture of both 3- and 8-glucuronides. Although we have the corresponding pure standards, both isomers display the same chromatographic and UV and MS spectroscopic features. Uro-A was poorly bioavailable, and trace amounts (below LOQ) were detected in all the animals, which prevented the quantitative comparison of plasma Uro-A levels between LPS-treated and control rats.

When LPS was given after $3 \mathrm{~h}$ of oral Uro-A administration, a significant increase in circulating Uro-A glur was observed (Fig. 1) in comparison with control rats despite the variability detected. The pharmacokinetic analysis revealed a significant increase of $C_{\max }$ and $\mathrm{AUC}_{\text {last }}$ in LPS-treated vs. control rats (Table 1). No effect of LPS was observed in the circulating levels of Uro-A sul (ESI Fig. $3 \dagger$ and Table 1).
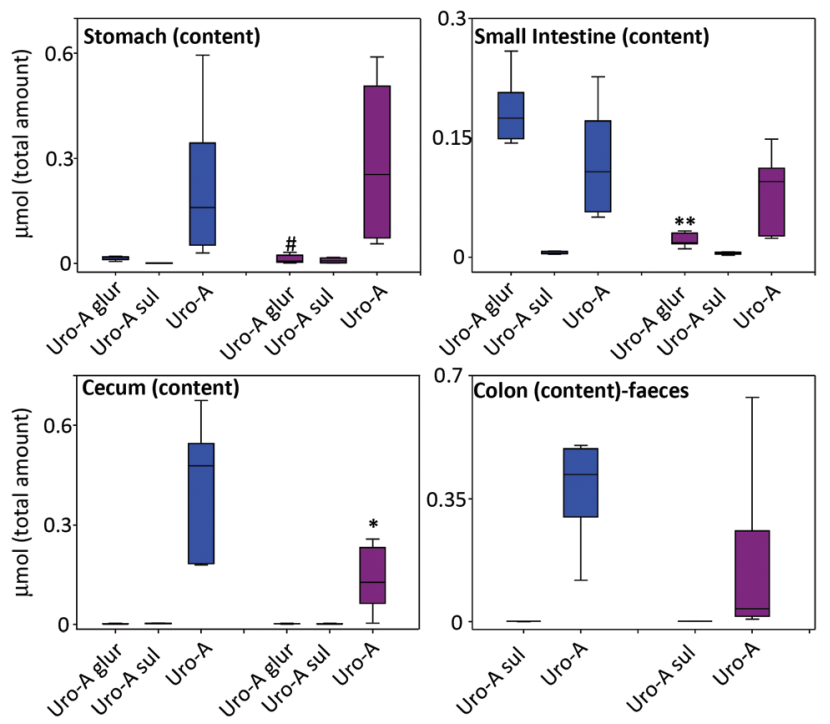

Fig. 2 Distribution of Uro-A and derived metabolites in the rat gastrointestinal contents. (Colon content and fresh stool showed similar values and are presented jointly.) Mean values \pm SD are shown. $\square$ Control; LPS-treated rats. $0.1>{ }^{\#} P>0.05 ;{ }^{*} P<0.05 ;{ }^{*} P<0.01$.

\section{Effect of LPS on the distribution of Uro-A and derived metabolites in the gastrointestinal tract}

At sacrifice, after $8 \mathrm{~h}$ of oral Uro-A administration, there was a tendency towards a lower amount of Uro-A and Uro-A glur in the gastrointestinal tract of LPS-treated $v s$. control rats, which became statistically significant in the case of Uro-A glur in the small intestine and Uro-A in the cecum (Fig. 2).

\section{Effect of LPS on the tissue distribution and urinary excretion of Uro-A and derived metabolites}

In the tissues, the amount of Uro-A and derived metabolites was higher in LPS-treated vs. control rats (Fig. 3). The most relevant results were observed in the liver, bladder, lung and spleen tissues where a significant deconjugation of Uro-A glur to Uro-A occurred upon LPS treatment. The amount of free Uro-A detected in the spleen and lungs was roughly equivalent to $2 \mu \mathrm{M}$. In contrast, a low and similar amount of metabolites was detected in the prostate (results not shown).

Table 1 Pharmacokinetics of Uro-A-derived metabolites after oral Uro-A administration in the control and LPS-treated rats

\begin{tabular}{|c|c|c|c|c|c|c|}
\hline & $T_{\max }(\mathrm{h})$ & $C_{\max }(\mu \mathrm{M})$ & $T_{\text {last }}(\mathrm{h})$ & $C_{\text {last }}(\mu \mathrm{M})$ & $\begin{array}{l}\text { AUC }_{\text {last }} \\
\left(\mu \mathrm{mol} \mathrm{h} \mathrm{L}^{-1}\right)\end{array}$ & $\begin{array}{l}\text { MRT }_{\text {last }} \\
\text { (h) }\end{array}$ \\
\hline \multicolumn{7}{|l|}{ Uro-A glur } \\
\hline Control & $4.0 \pm 2.4$ & $0.5 \pm 0.0$ & $8.0 \pm 0.0$ & $0.5 \pm 0.0$ & $3.2 \pm 0.4$ & $4.5 \pm 0.4$ \\
\hline LPS-treated & $5.8 \pm 2.3^{\#}$ & $1.3 \pm 0.5^{* * *}$ & $7.5 \pm 1.2$ & $1.2 \pm 0.5^{* *}$ & $4.9 \pm 1.5^{*}$ & $4.7 \pm 0.8$ \\
\hline \multicolumn{7}{|l|}{ Uro-A sul } \\
\hline Control & $5.4 \pm 3.1$ & $0.1 \pm 0.1$ & $8.0 \pm 0.0$ & $0.1 \pm 0.0$ & $0.5 \pm 0.1$ & $4.7 \pm 0.5$ \\
\hline LPS-treated & $5.3 \pm 2.1$ & $0.1 \pm 0.0$ & $7.2 \pm 1.2$ & $0.1 \pm 0.0$ & $0.4 \pm 0.1$ & $4.2 \pm 0.7$ \\
\hline
\end{tabular}

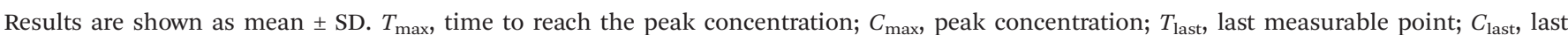

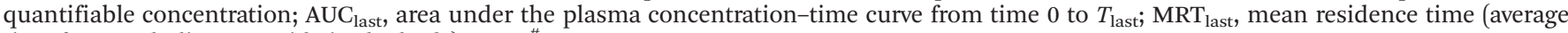
time for metabolites to reside in the body). $0.1>{ }^{\sharp} P>0.05 ;{ }^{*} P<0.05 ;{ }^{*} P<0.01 ;{ }^{* *} P<0.001$. 

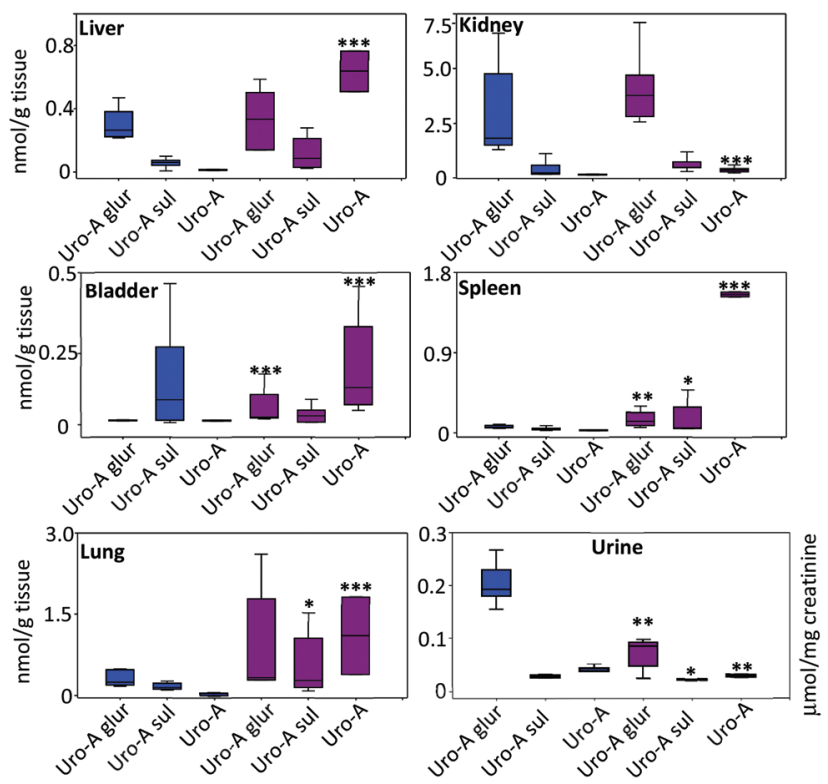

Fig. 3 Distribution of Uro-A and derived metabolites in tissues and urine. Mean values \pm SD are shown. $\square$ Control; $\square$ LPS-treated rats.

Regarding the urinary excretion, in contrast to the profile observed in tissues, there was a significant decrease of free Uro-A, Uro-A glur and Uro-A sul in LPS-treated vs. control rats (Fig. 3). Uro-A diglucuronide and Uro-A sulphoglucuronide were also identified but not quantified due to the lack of standards. The urinary excretion of these metabolites (peak area) was similar in both groups (results not shown).

The values of the Uro-A glur/Uro-A ratios highlighted the deconjugation process. The ratios were higher in the control than in the LPS-treated rats with the exception of the stomach and cecum contents, reaching statistical significance in all the organs and reservoirs except in the stomach and cecum (Table 2).

Table 2 Comparison of the Uro-A glur/Uro-A ratio in the control vs. LPS-treated rats

\begin{tabular}{llll}
\hline & Control & LPS & $P$-Value \\
\hline Stomach & $0.08 \pm 0.05$ & $0.11 \pm 0.10$ & 0.740 \\
Small intestine & $1.21 \pm 0.34$ & $0.32 \pm 0.21$ & $\mathbf{0 . 0 2 1}$ \\
Cecum & $0.02 \pm 0.01$ & $0.21 \pm 0.13$ & 0.150 \\
Colon & ND & ND & ND \\
Liver & $227 \pm 89$ & $0.45 \pm 0.04$ & $\mathbf{0 . 0 0 1}$ \\
Kidney & $2050 \pm 1100$ & $13.30 \pm 7.41$ & $\mathbf{0 . 0 0 2}$ \\
Bladder & $6.20 \pm 0.45$ & $0.22 \pm 0.19$ & $\mathbf{0 . 0 0 1}$ \\
Spleen & $25.7 \pm 11.2$ & $0.04 \pm 0.02$ & $\mathbf{0 . 0 0 4}$ \\
Lung & $127.4 \pm 19.9$ & $0.81 \pm 0.12$ & $\mathbf{0 . 0 0 1}$ \\
Urine & $4.98 \pm 1.37$ & $3.18 \pm 0.83$ & $\mathbf{0 . 0 3 4}$
\end{tabular}

Ratios are shown as mean \pm SD. Significant differences $(P<0.05)$ between the control and LPS ratios are bolded. ND, not determined (Uro-A glur was below the quantitation limit).

\section{Discussion}

We show here for the first time the tissue deconjugation of Uro-A glur to free Uro-A after oral Uro-A administration in a rat model of LPS-induced systemic inflammation. Our results provide further evidence on the 'deconjugation in inflammation' hypothesis, in agreement with previous reports on other phenolics. ${ }^{25-32}$

Urolithins represent a link between diet, gut microbiota and health, which has attracted increasing interest. ${ }^{1,5}$ Many in vitro studies have described a wide range of activities for these molecules in the last decade, especially anti-inflammatory and cancer chemopreventive activities. However, most of these studies are far from representing physiologically relevant conditions. ${ }^{1,38}$ The general pitfall is the assay of free urolithins, usually at high concentration, in cells from systemic organs instead of assaying the corresponding conjugates (glucuronides, sulphates, etc.), which are the molecules that can reach the systemic tissues. In fact, this concern is common for all dietary polyphenols. ${ }^{38}$

The anti-inflammatory activity and preservation of the barrier integrity of Uro-A in the colon ${ }^{7,8}$ are properties consistent with the high concentrations reached by Uro-A in the colon. ${ }^{9}$ However, the metabolites responsible for the systemic effects observed after the oral administration of Uro- $\mathrm{A}^{10,39}$ have not yet been described.

Uro-A is actively glucuronidated in the enterocyte before entering the bloodstream as Uro-A glur. ${ }^{5}$ Remarkably, Uro-A but not Uro-A glur has been reported to be a substrate of the Breast Cancer Resistant Protein (ABCG2/BCRP), ${ }^{40}$ a transporter that prevents specific molecules to enter the bloodstream and therefore restricts their distribution into organs. ${ }^{41}$ Therefore, the active glucuronidation and the basolateral-apical efflux of Uro-A by BCRP could explain the negligible concentration of Uro-A in the tissues (Fig. 2). ${ }^{11,12,40}$ Remarkably, the administration of LPS promoted a higher plasma Uro-A glur concentration $v s$. control rats (Fig. 1), which was consistent with the inhibition of ABC transporters by LPS. ${ }^{42}$ Therefore, although it may seem paradoxical at first glance, our results are compatible with the fact that LPS treatment favoured the delivery of Uro-A glur to the systemic tissues (inhibition of the BCR transporter), concomitant with a gradual release of free Uro-A from deconjugation by systemic inflammation (Fig. 3 and Table 2). The increase of glucuronidase activity in the systemic tissues of endotoxemic animals has been previously reported, ${ }^{43}$ which supports the glucuronidase-mediated deconjugation observed in our study. In a parallel scenario, LPS treatment increased benzo(a)pyreneinduced DNA adduct levels in the lung and liver tissues of benzo(a)pyrene inhalatory-exposed mice. ${ }^{44}$ This process was associated with the hydrolysis of benzo(a)pyrene glucuronide, which was further confirmed by the mechanistic studies of Shi et $a .^{45}$ In the present study, the deconjugation of Uro-A glur to free Uro-A was also supported by the increase of pro-inflammatory cytokines in systemic tissues (results not shown), in agreement with a previous study conducted under the same assay conditions. ${ }^{34}$ Although plasma $\beta$-glucuronidase also increased 
after LPS treatment (ESI Fig. $2 \dagger$ ), we could not detect the same deconjugation process in the bloodstream. We consider two hypotheses: (i) the low solubility of free Uro-A along with a quick distribution of Uro-A glur to systemic tissues prevented the quantification of this process in plasma, and(or) (ii) the potential adduct formation between Uro-A and blood proteins as reported for other phenolics and derivatives. ${ }^{46}$ Following the same rationale, we cannot discard a continuous in situ Uro-A glur to Uro-A deconjugation in systemic tissues, and promoting a possible accumulation of free Uro-A precipitates as a result of its high insolubility in aqueous matrixes. However, the confirmation of the above points requires further research. Finally, we also observed a lower urinary excretion of Uro-A and derived metabolites in endotoxemic rats, which was compatible with the LPS-induced renal failure, a process that can be evident after 3 hours of LPS administration. ${ }^{47}$

In the case of the flavonoid quercetin, the deconjugation of quercetin-glucuronide to quercetin has been reported to be mediated by mitochondrial dysfunction in inflammatory macrophages with a relevant deconjugation in the spleen, ${ }^{30}$ which agreed with our results in the case of Uro-A (Fig. 3). Besides, a possible increase of Uro-A, mediated by the deconjugation in circulating macrophages, could also explain the low levels of free Uro-A in plasma samples (i.e., devoid of cells and proteins after acetonitrile precipitation). Although we still do not know the exact mechanisms behind the tissue deconjugation of Uro-A glur to Uro-A, there is a well-known interplay between inflammatory stimuli, mitophagy and promotion/ inhibition of inflammation ${ }^{48,49}$ that could be involved in the deconjugation process of Uro-A glur to Uro-A, and in agreement with the studies on quercetin. ${ }^{30}$

\section{Conclusions}

We show here for the first time the inflammatory-mediated tissue deconjugation of Uro-A glur to Uro-A. These results contribute to consolidating the hypothesis of deconjugation in inflammation in the case of urolithins. We are aware that more research is needed to unravel the precise mechanisms underlying the deconjugation process in urolithins. However, our results show that endotoxemia increases free Uro-A in systemic tissues, reaching relevant concentrations, and compatible with in vitro specific biological activities attributed to free Uro-A. The ongoing research in our laboratory will shed some light on this process in the near future.

\section{Conflicts of interest}

There are no conflicts to declare.

\section{Acknowledgements}

This research has been supported by the Project 201770E081 from the Spanish National Research Council (CSIC, Spain) and by the Project AGL2015-64124-R (Ministry of Science, Innovation and Universities, Spain). J. A. G.-B. is a holder of a "Juan de la Cierva" contract from the Ministry of Science, Innovation and Universities (Spain).

\section{References}

1 F. A. Tomás-Barberán, A. González-Sarrías, R. GarcíaVillalba, M. A. Núñez-Sánchez, M. V. Selma, M. T. GarcíaConesa and J. C. Espín, Urolithins, the rescue of "old" metabolites to understand a "new" concept: Metabotypes as a nexus among phenolic metabolism, microbiota dysbiosis, and host health status, Mol. Nutr. Food Res., 2017, 61, 1500901.

2 J. Heilman, P. Andreux, N. Tran, C. Rinsch and W. BlancoBose, Safety assessment of Urolithin A, a metabolite produced by the human gut microbiota upon dietary intake of plant derived ellagitannins and ellagic acid, Food Chem. Toxicol., 2017, 108, 289-297.

3 A. Cortés-Martín, R. García-Villalba, A. González-Sarrías, M. Romo-Vaquero, V. Loria-Kohen, A. Ramírez-de-Molina, F. A. Tomás-Barberán, M. V. Selma and J. C. Espín, The gut microbiota urolithin metabotypes revisited: the human metabolism of ellagic acid is mainly determined by aging, Food Funct., 2018, 9, 4100-4106.

4 B. Cerdá, F. A. Tomás-Barberán and J. C. Espín, Metabolism of antioxidant and chemopreventive ellagitannins from strawberries, raspberries, walnuts, and oak-aged wine in humans: identification of biomarkers and individual variability, J. Agric. Food Chem., 2005, 53, 227-235.

5 J. C. Espín, M. Larrosa, M. T. García-Conesa and F. TomásBarberán, Biological significance of urolithins, the gut microbial ellagic Acid-derived metabolites: the evidence so far, Evid. Based Complement. Alternat. Med., 2013, 2013, 270418.

6 A. González-Sarrías, R. García-Villalba, M. Romo-Vaquero, C. Alasalvar, A. Örem, P. Zafrilla, F. A. Tomás-Barberán, M. V. Selma and J. C. Espín, Clustering according to urolithin metabotype explains the interindividual variability in the improvement of cardiovascular risk biomarkers in overweight-obese individuals consuming pomegranate: A randomized clinical trial, Mol. Nutr. Food Res., 2017, 61, 1600830.

7 M. Larrosa, A. González-Sarrías, M. J. Yáñez-Gascón, M. V. Selma, M. Azorín-Ortuño, S. Toti, F. Tomás-Barberán, P. Dolara and J. C. Espín, Anti-inflammatory properties of a pomegranate extract and its metabolite urolithin-A in a colitis rat model and the effect of colon inflammation on phenolic metabolism, J. Nutr. Biochem., 2010, 21, 717-725.

8 R. Singh, S. Chandrashekharappa, S. R. Bodduluri, B. V. Baby, B. Hegde, N. G. Kotla, A. A. Hiwale, T. Saiyed, P. Patel, M. Vijay-Kumar, M. G. I. Langille, G. M. Douglas, X. Cheng, E. C. Rouchka, S. J. Waigel, G. W. Dryden, H. Alatassi, H. G. Zhang, B. Haribabu, P. K. Vemula and V. R. Jala, Enhancement of the gut barrier integrity by a 
microbial metabolite through the Nrf2 pathway, Nat. Commun., 2019, 10(1), 89.

9 M. A. Nuñez-Sánchez, R. García-Villalba, T. Monedero-Saiz, N. V. García-Talavera, M. B. Gómez-Sánchez, C. SánchezÁlvarez, A. M. García-Albert, F. J. Rodríguez-Gil, M. RuizMarín, F. A. Pastor-Quirante, F. Martínez-Díaz, M. J. YáñezGascón, A. González-Sarrías, F. A. Tomás-Barberán and J. C. Espín, Targeted metabolic profiling of pomegranate polyphenols and urolithins in plasma, urine and colon tissues from colorectal cancer patients, Mol. Nutr. Food Res., 2014, 58, 1199-1211.

10 D. Ryu, L. Mouchiroud, P. A. Andreux, E. Katsyuba, N. Moullan, A. A. Nicolet-Dit-Félix, E. G. Williams, P. Jha, G. Lo Sasso, D. Huzard, P. Aebischer, C. Sandi, C. Rinsch and J. Auwerx, Urolithin A induces mitophagy and prolongs lifespan in C. elegans and increases muscle function in rodents, Nat. Med., 2016, 22, 879-888.

11 A. González-Sarrías, M. Azorín-Ortuño, M. J. Yáñez-Gascón, F. A. Tomás-Barberán, M. T. García-Conesa and J. C. Espín, Dissimilar in vitro and in vivo effects of ellagic acid and its microbiota-derived metabolites, urolithins, on the cytochrome P450 1A1, J. Agric. Food Chem., 2009, 57, 56235632.

12 A. González-Sarrías, J. A. Giménez-Bastida, M. T. GarcíaConesa, M. B. Gómez-Sánchez, N. V. García-Talavera, A. GilIzquierdo, C. Sánchez-Alvarez, L. O. Fontana-Compiano, J. P. Morga-Egea, F. A. Pastor-Quirante, F. Martínez-Díaz, F. A. Tomás-Barberán and J. C. Espín, Occurrence of urolithins, gut microbiota ellagic acid metabolites and proliferation markers expression response in the human prostate gland upon consumption of walnuts and pomegranate juice, Mol. Nutr. Food Res., 2010, 54, 311-322.

13 J. C. Espín, R. González-Barrio, B. Cerdá, C. López-Bote, A. I. Rey and F. A. Tomás-Barberán, Iberian pig as a model to clarify obscure points in the bioavailability and metabolism of ellagitannins in humans, J. Agric. Food Chem., 2007, 55(25), 10476-10485.

14 J. A. Giménez-Bastida, A. González-Sarrías, M. Larrosa, F. Tomás-Barberán, J. C. Espín and M. T. García-Conesa, Ellagitannin metabolites, urolithin A glucuronide and its aglycone urolithin A, ameliorate TNF- $\alpha$-induced inflammation and associated molecular markers in human aortic endothelial cells, Mol. Nutr. Food Res., 2012, 56, 784-796.

15 A. González-Sarrías, J. A. Giménez-Bastida, M. Á. NúñezSánchez, M. Larrosa, M. T. García-Conesa, F. A. TomásBarberán and J. C. Espín, Phase-II metabolism limits the antiproliferative activity of urolithins in human colon cancer cells, Eur. J. Nutr., 2014, 53, 853-864.

16 M. Á. Ávila-Gálvez, J. C. Espín and A. González-Sarrías, Physiological relevance of the antiproliferative and estrogenic effects of dietary polyphenol aglycones versus their phase-II metabolites on breast cancer cells: A call of caution, J. Agric. Food Chem., 2018, 66, 8547-8555.

17 M. Á. Ávila-Gálvez, R. García-Villalba, F. Martínez-Díaz, B. Ocaña-Castillo, T. Monedero-Saiz, A. Torrecillas-Sánchez, B. Abellán, A. González-Sarrías and J. C. Espín, Metabolic
Profiling of Dietary Polyphenols and Methylxanthines in Normal and Malignant Mammary Tissues from Breast Cancer Patients, Mol. Nutr. Food Res., 2019, e1801239.

18 M. Monagas, M. Urpi-Sarda, F. Sánchez-Patán, R. Llorach, I. Garrido, C. Gómez-Cordovés, C. Andres-Lacueva and B. Bartolomé, Insights into the metabolism and microbial biotransformation of dietary flavan-3-ols and the bioactivity of their metabolites, Food Funct., 2010, 1, 233-253.

19 J. Tomé-Carneiro, M. Larrosa, A. González-Sarrías, F. A. Tomás-Barberán, M. T. García-Conesa and J. C. Espín, Resveratrol and clinical trials: the crossroad from in vitro studies to human evidence, Curr. Pharm. Des., 2013, 19, 6064-6093.

20 T. Bohn, G. J. McDougall, A. Alegría, M. Alminger, E. Arrigoni, A. M. Aura, C. Brito, A. Cilla, S. N. El, S. Karakaya, M. C. Martínez-Cuesta and C. N. Santos, Mind the gap-deficits in our knowledge of aspects impacting the bioavailability of phytochemicals and their metabolites-a position paper focusing on carotenoids and polyphenols, Mol. Nutr. Food Res., 2015, 59, 1307-1323.

21 F. A. Tomás-Barberán, M. V. Selma and J. C. Espín, Interactions of gut microbiota with dietary polyphenols and consequences to human health, Curr. Opin. Clin. Nutr. Metab. Care, 2016, 19, 471-476.

22 F. Lodi, R. Jimenez, L. Moreno, P. A. Kroon, P. W. Needs, D. A. Hughes, C. Santos-Buelga, A. Gonzalez-Paramas, A. Cogolludo, R. Lopez-Sepulveda, J. Duarte and F. PerezVizcaino, Glucuronidated and sulfated metabolites of the flavonoid quercetin prevent endothelial dysfunction but lack direct vasorelaxant effects in rat aorta, Atherosclerosis, 2009, 204, 34-39.

23 K. R. Patel, C. Andreadi, R. G. Britton, E. Horner-Glister, A. Karmokar, S. Sale, V. A. Brown, D. E. Brenner, R. Singh, W. P. Steward, A. J. Gescher and K. Brown, Sulfate metabolites provide an intracellular pool for resveratrol generation and induce autophagy with senescence, Sci. Transl. Med., 2013, 5, 205ra133.

24 E. Van Rymenant, C. Grootaert, K. Beerens, P. W. Needs, P. A. Kroon, A. Kerimi, G. Williamson, R. García-Villalba, A. González-Sarrías, F. Tomas-Barberan, J. Van Camp and J. Van de Voorde, Vasorelaxant activity of twenty-one physiologically relevant (poly)phenolic metabolites on isolated mouse arteries, Food Funct., 2017, 8, 4331-4335.

25 K. Shimoi, N. Saka, K. Kaji, R. Nozawa and N. Kinae, Metabolic fate of luteolin and its functional activity at focal site, Biofactors, 2000, 12, 181-186.

26 K. Shimoi and T. Nakayama, Glucuronidase deconjugation in inflammation, Methods Enzymol., 2005, 400, 263-272.

27 A. G. Kunihiro, J. A. Brickey, J. B. Frye, P. B. Luis, C. Schneider and J. L. Funk, Curcumin, but not curcuminglucuronide, inhibits Smad signaling in TGF $\beta$-dependent bone metastatic breast cancer cells and is enriched in bone compared to other tissues, J. Nutr. Biochem., 2019, 63, 150156.

28 C. Menendez, M. Dueñas, P. Galindo, S. GonzálezManzano, R. Jimenez, L. Moreno, M. J. Zarzuelo, 
I. Rodríguez-Gómez, J. Duarte, C. Santos-Buelga and F. Perez-Vizcaino, Vascular deconjugation of quercetin glucuronide: the flavonoid paradox revealed?, Mol. Nutr. Food Res., 2011, 55, 1780-1790.

29 P. Galindo, I. Rodriguez-Gómez, S. González-Manzano, M. Dueñas, R. Jiménez, C. Menéndez, F. Vargas, J. Tamargo, C. Santos-Buelga, F. Pérez-Vizcaíno and J. Duarte, Glucuronidated quercetin lowers blood pressure in spontaneously hypertensive rats via deconjugation, PLoS One, 2012, 7, e32673.

30 A. Ishisaka, K. Kawabata, S. Miki, Y. Shiba, S. Minekawa, T. Nishikawa, R. Mukai, J. Terao and Y. Kawai, Mitochondrial dysfunction leads to deconjugation of quercetin glucuronides in inflammatory macrophages, PLoS One, 2013, 8, e80843.

31 J. Terao, Factors modulating bioavailability of quercetinrelated flavonoids and the consequences of their vascular function, Biochem. Pharmacol., 2017, 139, 15-23.

32 Y. Kawai, Understanding metabolic conversions and molecular actions of flavonoids in vivo: toward new strategies for effective utilization of natural polyphenols in human health, J. Med. Invest., 2018, 65, 162-165.

33 R. García-Villalba, J. C. Espín and F. A. Tomás-Barberán, Chromatographic and spectroscopic characterization of urolithins for their determination in biological samples after the intake of foods containing ellagitannins and ellagic acid, J. Chromatogr. A, 2016, 1428, 162-175.

34 M. Larrosa, M. Azorín-Ortuño, M. J. Yañez-Gascón, M. T. García-Conesa, F. Tomás-Barberán and J. C. Espín, Lack of effect of oral administration of resveratrol in LPSinduced systemic inflammation, Eur. J. Nutr., 2011, 50, 673-680.

35 S. Reagan-Shaw, M. Nihal and N. Ahmad, Dose translation from animal to human studies revisited, FASEB J., 2008, 22, 659-661.

36 M. Azorín-Ortuño, M. J. Yáñez-Gascón, F. Vallejo, F. J. Pallarés, M. Larrosa, R. Lucas, J. C. Morales, F. A. Tomás-Barberán, M. T. García-Conesa and J. C. Espín, Metabolites and tissue distribution of resveratrol in the pig, Mol. Nutr. Food Res., 2011, 55, 1154-1168.

37 Y. Zhang, M. Huo, J. Zhou and S. Xie, PKSolver: An add-in program for pharmacokinetic and pharmacodynamic data analysis in Microsoft Excel, Comput. Methods Programs Biomed., 2010, 99, 306-314.

38 M. Á. Ávila-Gálvez, A. González-Sarrías and J. C. Espín, In vitro research on dietary polyphenols and health: A call of caution and a guide on how to proceed, J. Agric. Food Chem., 2018, 66, 7857-7858.

39 H. Liu, H. Kang, C. Song, Z. Lei, L. Li, J. Guo, Y. Xu, H. Guan, Z. Fang and F. Li, Urolithin A inhibits the catabolic effect of TNF $\alpha$ on nucleus pulposus cell and alleviates intervertebral disc degeneration in vivo, Front. Pharmacol., 2018, 9, 1043.

40 A. González-Sarrías, V. Miguel, G. Merino, R. Lucas, J. C. Morales, F. Tomás-Barberán, A. I. Alvarez and J. C. Espín, The gut microbiota ellagic acid-derived metabolite urolithin A and its sulfate conjugate are substrates for the drug efflux transporter breast cancer resistance protein (ABCG2/BCRP), J. Agric. Food Chem., 2013, 61(18), 43524359.

41 M. Dean, Y. Hamon and G. Chimini, The human ATPbinding cassette (ABC) transporter superfamily, J. Lipid Res., 2001, 42(7), 1007-1017.

42 J. Kalitsky-Szirtes, A. Shayeganpour, D. R. Brocks and M. Piquette-Miller, Suppression of drug-metabolizing enzymes and efflux transporters in the intestine of endotoxin-treated rats, Drug Metab. Dispos., 2004, 32, 20-27.

43 E. P. Schmidt, Y. Yang, W. J. Janssen, A. Gandjeva, M. J. Perez, L. Barthel, R. L. Zemans, J. C. Bowman, D. E. Koyanagi, Z. X. Yunt, L. P. Smith, S. S. Cheng, K. H. Overdier, K. R. Thompson, M. W. Geraci, I. S. Douglas, D. B. Pearse and R. M. Tuder, The pulmonary endothelial glycocalyx regulates neutrophil adhesion and lung injury during experimental sepsis, Nat. Med., 2012, 18, 1217-1223.

44 V. M. Arlt, A. M. Krais, R. W. Godschalk, Y. Riffo-Vasquez, I. Mrizova, C. A. Roufosse, C. Corbin, Q. Shi, E. Frei, M. Stiborova, F. J. van Schooten, D. H. Phillips and D. Spina, Pulmonary inflammation impacts on CYP1A1mediated respiratory tract DNA damage induced by the carcinogenic air pollutant benzo[a]pyrene toxicological sciences, Toxicol. Sci., 2015, 146, 213-225.

45 Q. Shi, G. R. Haenen, L. Maas, V. M. Arlt, D. Spina, Y. R. Vasquez, E. Moonen, C. Veith, F. J. van Schooten and R. W. L. Godschalk, Inflammation-associated extracellular $\beta$-glucuronidase alters cellular responses to the chemical carcinogen benzo[a]pyrene, Arch. Toxicol., 2016, 90, 22612273.

46 R. L. Edwards, P. B. Luis, P. V. Varuzza, A. I. Joseph, S. H. Presley, R. Chaturvedi and C. Schneider, The antiinflammatory activity of curcumin is mediated by its oxidative metabolites, J. Biol. Chem., 2017, 292, 21243-21252.

47 M. Han, Y. Li, M. Liu, Y. Li and B. Cong, Renal neutrophil gelatinase associated lipocalin expression in lipopolysaccharide-induced acute kidney injury in the rat, $B M C$ Nephrol., 2012, 13, 25.

48 K. Minton, Inflammasome: Anti-inflammatory effect of mitophagy, Nat. Rev. Immunol., 2016, 16(4), 206.

49 Y. Zhao, S. Huang, J. Liu, X. Wu, S. Zhou, K. Dai and Y. Kou, Mitophagy contributes to the pathogenesis of inflammatory diseases, Inflammation, 2018, 41(5), 1590-1600. 